\section{Artificial Intelligence}

\section{B. Humpert, Indianapolis and Zurich}

\author{
(Purdue University, IKOSS Research \& Development)
}

Since the earliest days of computer conception, there has been a small, steadily growing, community of scientists who pursued the goal of simulating human activities with computers, such as physical work with robots, vision, language, human thinking, and more recently even psychology [1]. The ever increasing processing speed and storage capacity of today's computers, as well as the practical applicability of several Al concepts, have in recent years led to an explosive expansion of the field. The finance-economic community provides almost unlimited financial resources, and universities are hastily trying to integrate a field which has long been ignored. Large corporations are currently building up their own $\mathrm{Al}$ teams; the EEC has spent 1500 and budgeted a further 1600 million ECU on ESPRIT (European Strategic Program for Research and Development in Information Technology); the US Department of Defense has initiated a $\$ 500$ million, 5-year strategic computing program; the three European computer companies BULL, ICL and SIEMENS have started the ECRC (European Computer-Industry Research Center); and so on [2].

It is now appropriate to ask ourselves what the reason for all these activities is, and where the potential in this field lies? The main purposes of this article are to consider these questions and give insight into some of the most exciting present-day developments towards the setting up of instruments to allow for DEDUCTIVE REASONING and to encapsulate KNOWLEDGE with computers. Besides these themes we shall also discuss QUALITATIVE PHYSICS/REASONING/CONTROL, and the developments regarding NEURONAL NETWORKS.

\section{Theorem Proving / Automated Reason- ing [3]}

One of the most important aspects in $\mathrm{Al}$ is LOGIC, in particular 1st-order predicate (or mathematical) logic, which allows us to establish interrelations between objects. Logic is obviously a far more general way of expressing concepts, features and interconnections than mathematics. Loosely speaking, mathematics is a sub-discipline of logic. It is therefore not surprising that $\mathrm{Al}$ can attack problems of a far more general nature, and even find a common means for their solution, in much the same way as human thinking is the basis of mathematics, management, artistic activities, every-day problem solving, etc. Now, 1st-order predicate logic is semi-decisive, which means that a (computer-) procedure can be set up to determine the truth of a logical formula, provided it is true. Using the same procedure to test a false formula does not lead to any result, as the computer will search forever. A situation known as semi-decisiveness will therefore arise when the search process is terminated after a long timeperiod, in which case we do not know whether a formula is false or whether the search process did not proceed long enough.

Theorem-provers are symbolic computer programs which can establish whether a logical formula $C$ (= conjecture) logically follows from a set of other logical formulae $A i$ (= axioms):

$$
A 1{ }^{\wedge} A 22^{\wedge} . . \wedge A n->C \text {. }
$$

The $A i$ are themselves logical formulae with the connectives:

$$
\begin{aligned}
& \text { ^(and), v (or), }-\rangle \text { (if-then), } \\
& \langle-\rangle \text { (if-and-only-if), }
\end{aligned}
$$

as well as the existential $(\exists)$ and universal $(\forall)$ quantifiers. Several theorem-provers have been developed to prove mathematical theorems, but they are also used in a variety of other domains, such as logic circuit design, program debugging/verification, real-time control, metamathematics, communication protocols, assembly language, chemical synthesis, diagnosis, robotics, expert-systems, etc., which has led to the new research area of Automated Reasoning.

\section{Expert-Systems [4]}

This field has been developed in the last ten years and is today on the verge of becoming an intellectual industry. The expert-systems (ESs), or knowledgebased systems as they are also known, store specialized knowledge of a narrow domain in a computer. Examples include knowledge of a medical field such as blood diseases, or how to diagnose and repair a car, or how to configure a computer based on a customer's requirements. ESs usually encode this knowledge in the form of IF-THEN rules

\section{IF Condition-1 AND

$$
\text { Condition-2 AND }
$$

THEN Statement

ESs are composed of a rule-base containing the rules of a particular knowledge domain, a data-base which stores the initial as well as derived facts (certified facts), an inference engine which derives new facts by applying rules on the stored facts, and an input/output facility. The inference engine allows for the chaining of rules since the derived facts of earlier rules can be used as the premise for a later rule, a process that can be repeated in successive steps. One distinguishes here between forward chaining which is a continuous derivation of new facts from old ones, and backward chaining which tries to certify a fact by going backwards to the premises of a rule. The number of existing ESs is currently in the hundreds, possibly thousands, and the list of applications is also long: business, management, medicine, banking/finance, teaching/education, manufacturing, telecommunications, etc.

The field of ESs is currently in the phase of commercialization and there have already been net gains of several tens of millions of dollars.

\section{Qualitative Reasoning [5]}

This is a relatively new Al field with the purpose of explaining how (complex) physical systems work. Qualitative physics predicts and explains the behaviour of mechanisms in qualitative terms. Its goals are:

(1) to be far more simple than classical physics and yet retain all the important properties (e.g. state, oscillation, gain, momentum) without involving the mathematics of continuously varying quantities and differential equations,

(2) to produce causal accounts of physical mechanisms that are easy to understand, and

(3) to provide the foundations for common sense models for the next generation of ESs.

The behaviour of physical systems can be described by the values of its variables (forces, velocities, pressures, etc.) at each moment in time. Such a description, although complete, fails to provide much insight into how the system functions. The enlightening concepts and distinctions are usually qualitative, but they are embedded within the much more complex framework establi- 
shed by continuous real-valued variables and differential equations. The initiator's proposal is to reduce the quantitative precision of the behavioural descriptions, but retain the crucial distinctions. Instead of continuous real-valued variables, each variable is described qualitatively - taking on only a small number of values, usually,+- , and 0 . The central modelling tool is then the qualitative differential equation, called a confluence. For example, the qualitative behaviour of a valve is expressed by the confluence: $\partial P+\partial A-\partial Q=0$ where $Q$ is the flow through the valve, $P$ is the pressure across the valve, $A$ is the area available for flow, and $\partial Q, \partial A$ and $\partial P$ represent their changes. The confluence represents multiple competing tendencies: the change in area positively influences flow rate and negatively influences pressure, the change in pressure positively influences flow rate, etc. The same variable can appear in many confluences and thus can be influenced in many different ways. The motivation for developing a qualitative approach stems from problems in psychology, education, $\mathrm{Al}$, and physics. Humans appear to use qualitative causal calculus in reasoning about the behaviour of their physical environment. Judging from their explanations, this calculus is quite different from the classical physics taught in classrooms. This raises questions as to what this "naive" physics is like, and how it helps us to reason about the world. Research in qualitative reasoning is at present in the development phase, although several interesting applications for hardware analysis, the modelling of a distillation-column and even financial risk assessment, have been completed or proposed [6].

\section{Neuronal Networks [7]}

The world's first information revolution occurred not with digital computers and silicon chips, but with neurons, the cells that make up the brain and nervous system of every sentient being. Researchers are still a long way from understanding how these natural computers work, but electronic neural models, developed by neurobiologists, physicists and mathematicians, have shown such promise that they are spawning a whole new computer discipline known variously as neural networking, connectionism, adaptive systems, and neurocomputing. The main new aspect behind work in this direction is "parallelism". If a large number of processing units are interconnected, one is immediately faced with the problem of how their interconnection is arranged. The organization of biological systems serves partially as a model.

Neuronal networks consist of many simple neurons that have densely parallel interconnections. The neurons communicate across the connections by means of "activations" and "inhibitions" - signals that excite or inhibit responses by the connected neurons. $A$ neuron can receive input from many neurons, and if the sum of these input signals exceeds a set threshold value, the neuron will "fire", producing an output signal of a certain value. This output is in turn relayed to other neurons. In simulation models of such systems, different neurons can be programmed to have different output values. In addition, the connection between every pair of neurons is assigned a "weight" or "connection strength" that modifies each output value, determining the actual value of each signal transmitted to attached neurons. The study of such networks and their practical use is at the forefront of present-day research, because they are expected to make massive parallelism possible and this, in turn, opens the door to highly computerintensive problems such as spokenlanguage and picture processing, self- teaching, learning, complex pattern matching, and many others.

\section{Conclusion}

What have we learnt? Al is at present in a state of explosive expansion, as we witnessed 15 years ago in high-energy physics. It tackles problems of a much wider spectrum than physics. It is inherently multi-disciplinary to a degree which is only matched in philosophy. Some of the fundamental issues are deductive reasoning, knowledge storage and processing, and the study of massive parallelism which is associated with an understanding of the nervous system, and ultimately — the brain.

\section{Acknowledgements}

I should like to thank S. + R. Gerber and P. Holley for their kind help in the preparation of this paper.

\section{REFERENCES}

[1] Barr A. and Feigenbaum E.A., The Handbook of Artificial Intelligence: $I+11+I I I$ (HeurisTech Press, Stanford CA) 1981; Humpert B., 'Artificial Intelligence: Impact on Pure and Applied Science', Helv. Phys. Acta 59 (1986) 1196.

[2] Hayes-Roth F., Knowledge-Based Expert Systems, IEEE Computer Society, October 1984, pp. 263.

[3] Wos L. et al., Automated Reasoning: Introduction and Application (Prentice Hall) 1984; Humpert B., 'Automated Reasoning', Künstliche Intelligenz, Eds. $\mathrm{H}$. Bunke and $\mathrm{H}$. Mey (Herbert Lang Verlag, Bern) 1987.

[4] Harmon P. and King D., Expert Systems: Artificial Intelligence in Business (John Wiley \& Sons) 1985; Humpert B., 'Knowledge Engineering with Expert Systems', Helv. Phys. Acta 59 (1986) 21.

[5] Bobrow D.G., Qualitative Reasoning (North Holland) 1984

[6] Kinoshita J. and Palevsky N.G., 'Computing with Neural Networks', High Technology May 1987.

[7] Humpert B. and Holley P., 'Expert Systems in Finance-Planning', IKOSS R\&D/105 August 1987.

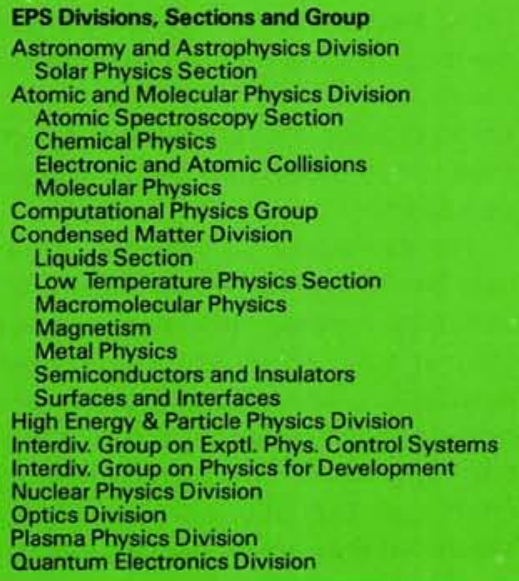

Europhysics News is the official journal of the European Europhysics News is the official journal of the European
Physical Society which comprises 29 National SociePhysical Society which comprises 29 National Socie-
ties, Academies and Group, about 4000 Individual Members and 75 Associate Members. Governing bodies of EPS are the General Meeting, Council and an elected Executive Committee responsible for detailed policy. EPS promotes the collaboration of physicists throughout Europe, organising and harmonising conferences and publications, improving physics education, encouraging physics applications, awarding scholarships to sponsored schools in Erice. EPS publishes in addition to Europhys. News, Europhysics Letters (in partnership with national societies), European Joumal of Physics (in partnership with The UK Inst. of Phys.), and European Conference Abstracts. Individual Members receive Europhys. News free of charge (price to insts. : Sw.Fr. 90.-la), Europhys. Lett. at Sw.Fr. 125.-/a (insts. $1050 .-$, rebates on many other publications and on conference fees. Annual EPS membership fee for Individual Members belonging to an EPS member society is: Sw.Fr. 44.-; independent members: Sw.Fr. 132.-; members of a Collaborating Society: Sw.Fr. 55.-

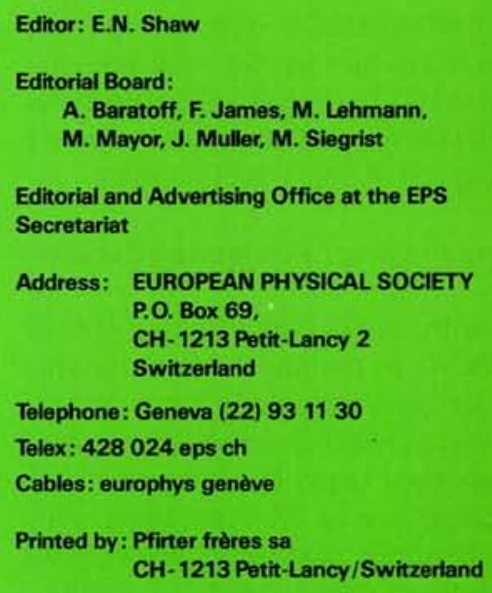

